\title{
Answer to Quiz, Discussion and Conclusion
}

\author{
CSP Sosai ${ }^{1}$, Anoj Dharmawardhane ${ }^{2}$ \\ 1. Department of Pathology, Colombo South Teaching Hospital, Sri Lanka \\ 2. Department of Surgery, Toowoomba Hospital, Queensland, Australia
}

\section{Answer to Quiz}

The histopathology of the jejunal lesions revealed a tumour in the submucosa (Fig2) with typical features of choriocarcinoma, comprising of a plexiform pattern of syncytiotrophoblasts and cytotrophoblasts amidst much haemorrhage (Fig. 3\&4).

The cytotrophoblastic cells appear sheet-like with large, bland, vesicular nuclei and prominent nucleoli. The cytoplasm is abundant and clear with sharp cytoplasmic margins. The syncitiotrophoblastic cells show the typical multinucleation with abundant, eosinophilic cytoplasm and are arranged around the cytotrophoblastic cells. Mitoses are many.

On retrospective inquiry, it was found that she had undergone evacuation of retained products of conception a year ago and the histopathology was a hydatidiform mole. She had defaulted follow up.

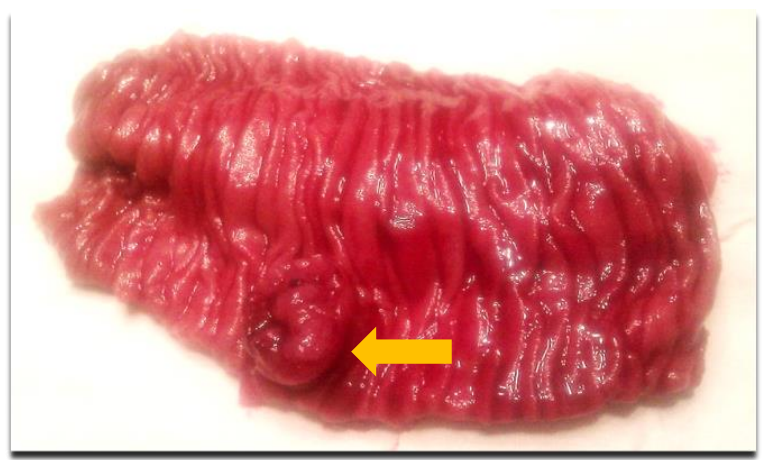

Figure.1. Polypoid tumour nodule in jejunum (yellow arrow)

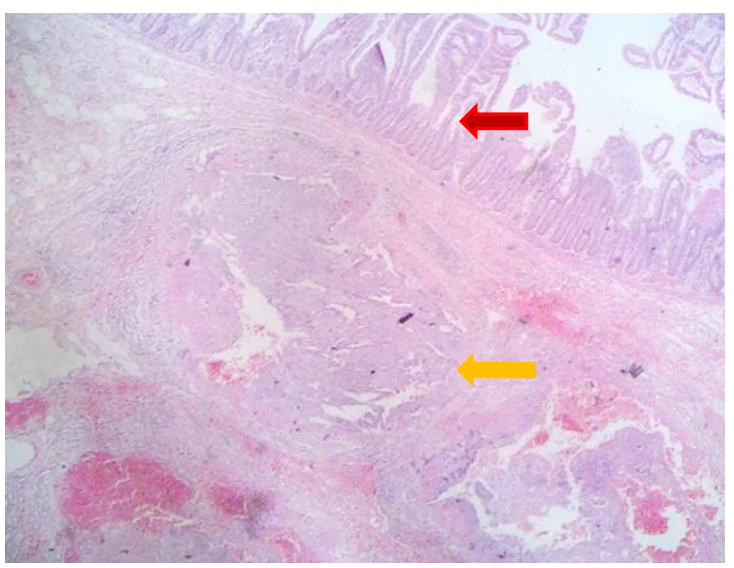

Figure 2: Jejunal mucosa (red arrow), submucosal deposit of choriocarcinoma (yellow arrow) (H\&EX5).

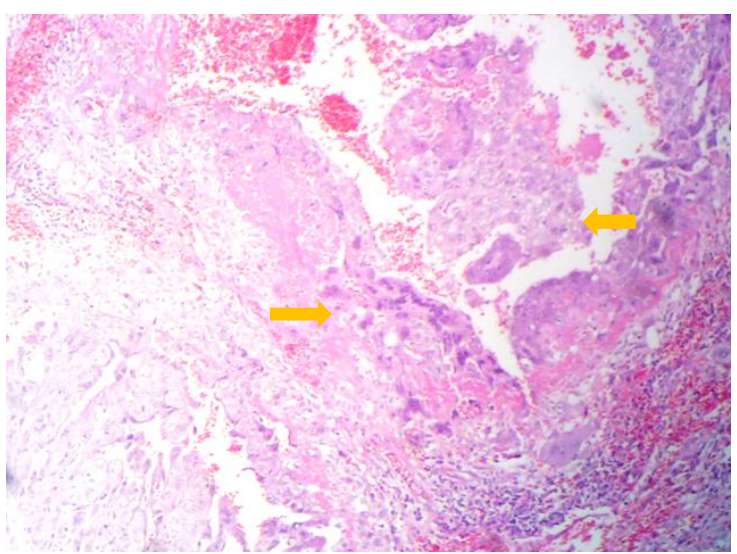

Figure 3: Plexiform pattern (arrows) (H\&EX10).

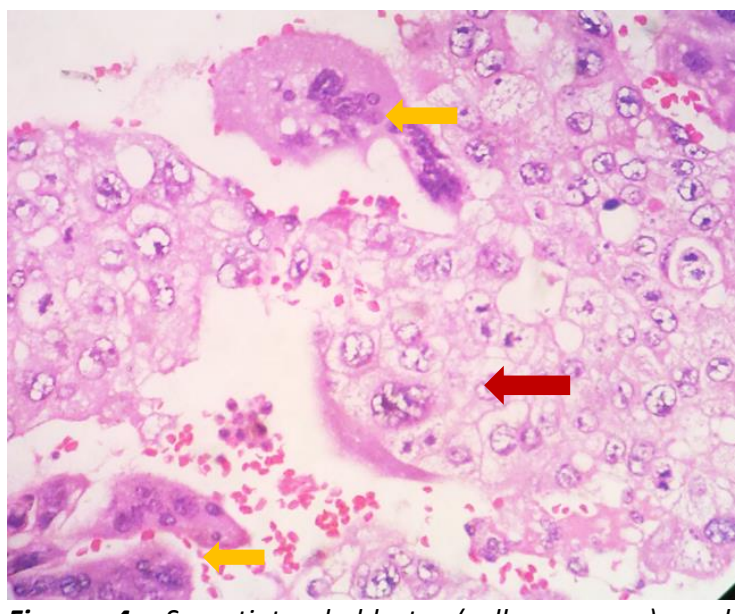

Figure 4: Syncytiotrophoblasts (yellow arrow) and cytotrophoblasts (red arrow) (H \&E X40) 


\section{Discussion}

Bleeding is an uncommon presentation of small intestinal tumours and only $10 \%$ of such patients are admitted with blood loss [1]. Causes of obscure bleeding necessitating laparoscopy or laparotomy for diagnosis include angiodysplasia, haemangioma, telangiectasia, Crohn disease, Meckel diverticulum, smooth muscle tumours, lymphoma and carcinoma. Primary tumours of the small bowel are rare and constitute about $5 \%$ of all tumors of the alimentary tract whereas metastatic tumours are seen frequently [1].

Deciphering the cause of hematochezia occurring in a young woman warrants several investigations and recognition of choriocarcinoma as its cause at an unexpected site is a challenge. Unless a relevant past history or clinical information is provided, the index of suspicion is the only key to go by to recognize this rapidly invasive metastasizing tumour lurking in on an unsuspecting patient and pathologist, since the source of haemorrhage may remain obscure despite exhaustive workup.

Intraoperative small bowel enteroscopy is a useful tool for the evaluation of small intestinal bleeding of obscure origin and angiography is most useful in the search for the site and cause of actively bleeding lesions [1].

Choriocarcinoma is a rapidly invasive, metastasizing tumour which is intrauterine and gestational. Primary or metastatic choriocarcinoma of the gastrointestinal tract is rare and only five percent of patients presenting with choriocarcinoma develop small bowel metastases [2,3]. They are seen commonly in the jejunum, followed by the duodenum and ileum, respectively $[2,4,5]$. Metastatic lesions of small intestine are often multiple and ulcerating [5].

Histopathological diagnosis of choriocarcinoma is straightforward when the typical mixture of cytotrophoblasts, that are polygonal with prominent cytoplasmic margins, clear cytoplasm, bland vesicular nucleus and syncytiotrophoblasts with multiple nuclei and abundant eosinophilic cytoplasm are present. The syncytiotrophoblasts cap around the cytotrophoblasts and are invariably associated with haemorrhage. Monophasic choriocarcinoma is very rare. It is important to distinguish a metastatic choriocarcinoma from a primary or metastatic poorly differentiated carcinoma or melanoma. Human chorionic gonadotrophin, human placental lactogen, glypican 3, inhibin, SALL4, beta catenin, GATA3 and p63 immuno markers are useful to identify choriocarcinoma. Melan A and HMB 45, CK7 and , CK 20 are useful markers in excluding a melanoma and carcinoma respectively. Most patients with a metastatic choriocarcinoma have an antecedent molar pregnancy but an associated choriocarcinomatous lesion in the uterus is absent in the majority, as in this case [6]. When there is no antecedent history, it is vital to differentiate gestational and nongestational origin of choriocarcinoma by documenting the presence of paternal alleles in gestational choriocarcinoma with short tandem repeat (STR) analysis [7]. The key factors for successful outcome is early diagnosis and initiation of appropriate therapy.

\section{References}

1.Lau WY, Fan ST, Wong SH, Wong KP, Poon GP, Chu KW, Yip WC, Wong KK, Preoperative and intraoperative localisation of gastrointestinal bleeding of obscure origin. Gut.1987; 28(7):869-77. doi: 10.1136/gut.28.7.869

2. M F Armellino et al. Jejunal perforation from metastatic choriocarcinoma. Case report and review of the literature. G Chir. (Italian Journal). 2008; 29(4):145-8.

3.Molina Infante J, Beceiro Pedreno I, Ripoll Noiseux C, et al. Gastrointestinal hemorrhage due to metastatic choriocarcinoma with gastric and colonic involvement. Rev Esp Enferm Dig. (Spanish Journal of gastroenterology). 2004; 96:77-80.

4. Yousefi Z, Mehrdizaj SG, Frimany, MB, et al. Metastatic choriocarcinoma in the small 
bowel: A case report. Tehran University Medical Journal. 2014; 72(5):335-8.

5. Yokoi K, Tanaka N, Furukawa K, et al. Male choriocarcinoma with metastasis to the jejunum: A case report and review of the literature. Journal of Nippon Medical School 2008; 75:115-7.

6. J Kumar, A llancheran, S S Ratnam. Pulmonary metastases in gestational trophoblastic disease: a review of 97 cases. British Journal of Obstetrics and Gynaecology. 1988 Jan; 95(1):70-4. doi: 10.1111/j.1471-0528. 1988.tb06482. x.

7. Johanna Savage et al. Choriocarcinoma in women: analysis of a case series with genotyping. American Journal of Surgical Pathology. 2017; 41(12):593.

DOI: 10.1097/PAS.0000000000000937 